\title{
Otolicuorrea espontánea en el adulto. Presentación de dos casos y revisión de la literatura
}

\author{
L. Galbarriatu*; J. Aurrecoechea*; E. Ruiz de Gopegui*; I. Pomposo*,****; G. Bilbao*,****; S. González*; J.I. \\ Undabeitia**; J.J. Novo**; K. Aguirrebengoa*** y J.M. Garibi****** \\ Servicios de Neurocirugía*, Otorrinolaringología** y ***Unidad de Enfermedades Infecciosas. Hospital Universitario de Cruces. \\ ****Universidad del País Vasco.
}

\section{Resumen}

Introducción. Las fístulas espontáneas de LCR no son una entidad frecuente $y$ pueden ser infradiagnosticadas en el adulto. Para algunos autores, debe sospecharse esta patología en cualquier paciente mayor de 50 años con episodios de otitis serosas recurrentes y ausencia de antecedentes otológicos. La meningitis es su complicación más grave, por lo que es prioritario un diagnóstico precoz y certero.

Objetivo. Presentar dos casos clínicos atendidos en nuestro servicio y revisar la literatura científica existente al respecto.

Conclusión El tratamiento de estas fístulas atraumáticas es eminentemente quirúrgico, pudiendo emplear la craneotomía de fosa media o la vía transmastoidea. En el sellado debe emplearse una técnica multicapa, combinando materiales autólogos y artificiales para conseguir mejores resultados.

PALABRAS CLAVE: Otolicuorrea espontánea. Fístula de LCR. Otitis serosa media. Defectos hueso temporal.

Adult spontaneous cerebrospinal fluid otorrhea. Report of two cases and review of the literature

\section{Summary}

Introduction. Spontaneous cerebrospinal fluid otorrhea is a relatively rare entity and can be easily missed in adults. Every adult older than 50 years with a negative history of otologic disease who has recurrent serous otitis media should be evaluated for this pathology. Meningitis is the most serious complication, so there is no doubt that the condition needs immediate attention and correction.

Objective. We present two patients who were diagnosed with spontaneous CSF otorrhea and make a review of what is reported about this topic.

Recibido: 10-06-10. Aceptado: 20-08-10
Conclusion. Surgical repair is mandatory to seal these nontraumatic CSF leaks. There are two main surgical approaches, the middle fossa craniotomy and the transmastoid approach. A multilayered closure technique in which autologous and artificial materials are combined is considered to result in the highest rate of success.

KEY WORDS: Spontaneous CSF otorrhea. CSF leak. Serous otitis media. Temporal bone defects.

\section{Introducción}

Las fístulas espontáneas de LCR a través del hueso temporal no son una entidad frecuente y pueden ser infradiagnosticadas en el adulto ${ }^{3}$. Escat recogió el primer caso al respecto en la literatura francesa en $1897^{15}$ y desde entonces, cada vez un mayor número de series analizan las peculiaridades de esta patología. A pesar de su rareza desde un punto de vista sintomático, diversos estudios en cadáver muestran defectos en el hueso temporal hasta en el 34\% de los casos, siendo múltiples en menos del $1 \%{ }^{3}$. La otolicuorrea espontánea es consecuencia de un defecto dural que causa una anormal comunicación entre el espacio subaracnoideo y las cavidades neumatizadas del hueso temporal ${ }^{17}$. Dicha comunicación constituye una vía de entrada de patógenos y la posibilidad de desarrollar una infección del sistema nervioso central, que es su complicación más grave ${ }^{3}$. El riesgo de meningitis en pacientes con fístula de LCR varía desde un 4 hasta un 50\% según las diversas causas ${ }^{18}$, y el tratamiento antibiótico no es efectivo en su profilaxis, como lo demuestran estudios previos. En general, la clínica de presentación suele ser insidiosa y poco específica, por lo que en el diagnóstico es fundamental una exhaustiva evaluación clínica y un alto índice de sospecha, dadas las graves consecuencias de un juicio erróneo ${ }^{6}$. Para algunos autores, en aquellos

Abreviaturas. LCR: líquido cefalorraquideo. CAE: conducto auditivo externo. 


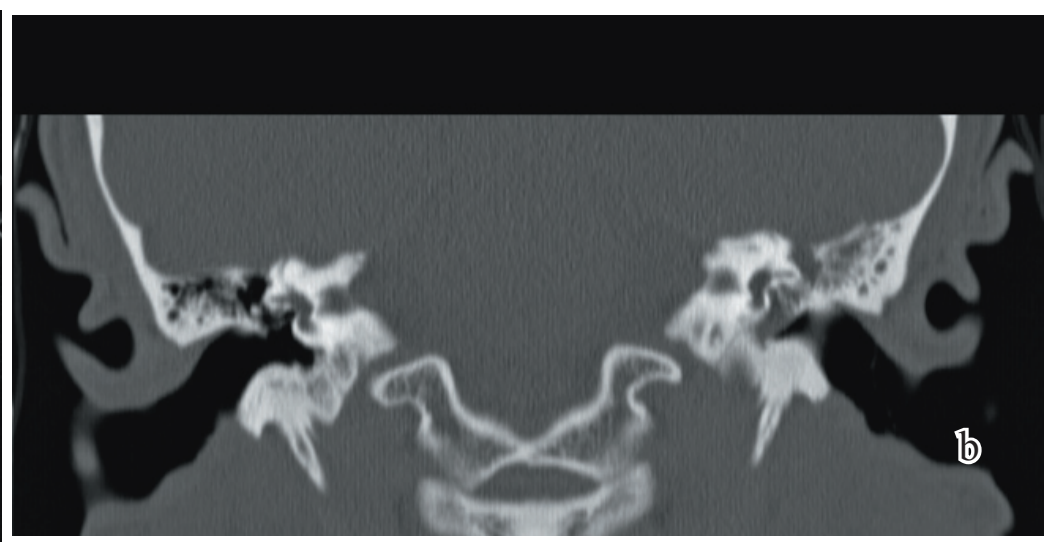

Figura 1. Imágenes de TAC axial y coronal que muestran: a) ocupación de celdillas mastoideas y cavidad timpánica del lado izquierdo y b) solución de continuidad del tegmen timpani del mismo lado.

pacientes mayores de 50 años sin historia de patología otológica, con episodios recurrentes de otitis serosa en oído medio, debe sospecharse una fístula de LCR hasta no demostrar lo contrario ${ }^{7}$. A pesar de todo, no existe un consenso en la literatura sobre el diagnóstico y manejo de esta entidad ${ }^{3}$.

A continuación, se presentan dos casos de otolicuorrea espontánea en pacientes adultos, así como una revisión de la clínica, diagnóstico y tratamiento de esta entidad.

\section{Caso clínico 1}

Varón de 37 años, con antecedentes de amigdalitis de repetición en la infancia y dolor frecuente de oídos. En su historia, no se refleja antecedente traumático. Debuta en el año 2006 con una meningitis neumocócica bacteriémica que requiere ingreso en UCI. En la TAC realizada durante el ingreso se observa una ocupación del oído medio, CAE y celdillas mastoideas izquierdas, sugestivo de otomastoiditis izquierda como origen de la infección. En seguimiento posterior por el servicio de Otorrinolaringología y ante una probable otitis seromucosa crónica, se coloca un drenaje transtimpánico en oído izquierdo, objetivando una otolicuorrea clara y persistente. El paciente presenta un segundo episodio de meningitis, y en la TAC cerebral y de peñascos realizada, se visualiza una solución de continuidad ósea en el techo timpánico y celdas mastoideas en el lado izquierdo (fig. 1), en probable relación con fístula de LCR. En la RM cerebral no se observa herniación de tejido cerebral a través del defecto. Se procede a realizar una craneotomía temporal izquierda, apreciándose un defecto único en el tegmen timpani, que se sella con hueso autólogo, fascia temporal, pegamento biológico y plastia dural artificial (fig. 2). El postoperatorio cursa sin incidencias, comprobando en la TAC postquirúrgica el cierre del defecto sin otros datos de complicación. Hasta la fecha, el paciente no ha vuelto a presentar síntomas.
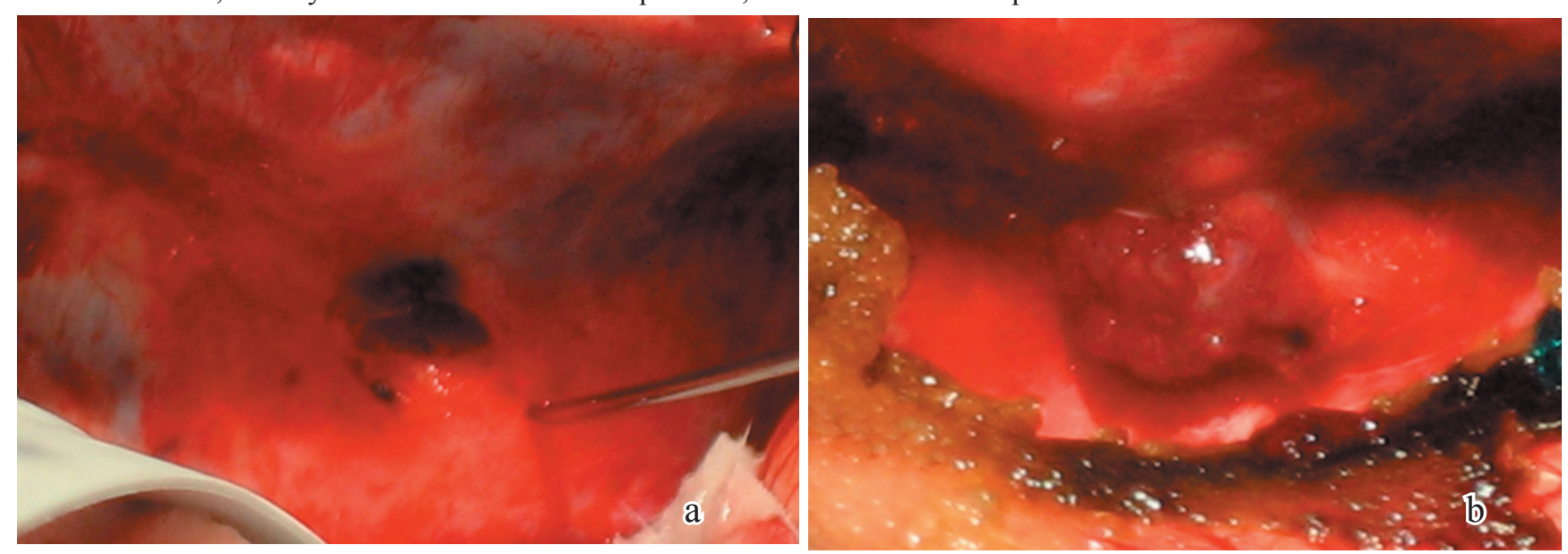

Figura 2. Imágenes intraoperatorias: a) defecto único en el tegmen timpani; b) reparación con hueso autólogo, fascia temporal, pegamento biológico y plastia dural artificial. 


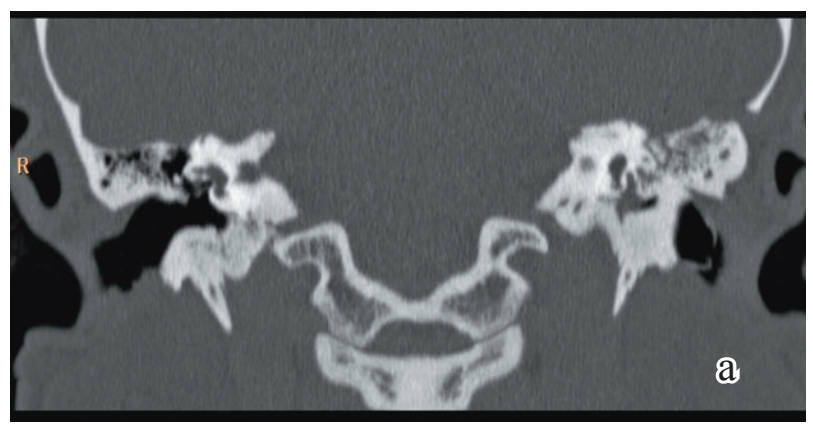

Figura 3: a) TAC postoperatoria, corte coronal. Se aprecia la reparación del defecto con fragmentos de hueso autólogo; b) Reconstrucción 3D mostrando el fragmento óseo (en círculo).

\section{Caso clínico 2}

Mujer de 39 años, fumadora y sin otros antecedentes de interés. Seguida en consultas de Otorrinolaringología por hipoacusia izquierda, con diagnóstico inicial de otitis serosa persistente. Tras realización de miringotomía y colocación de drenaje transtimpánico en el oído izquierdo, presenta otolicuorrea clara, y la TAC dirigida a peñascos pone de manifiesto una interrupción del techo del tímpano

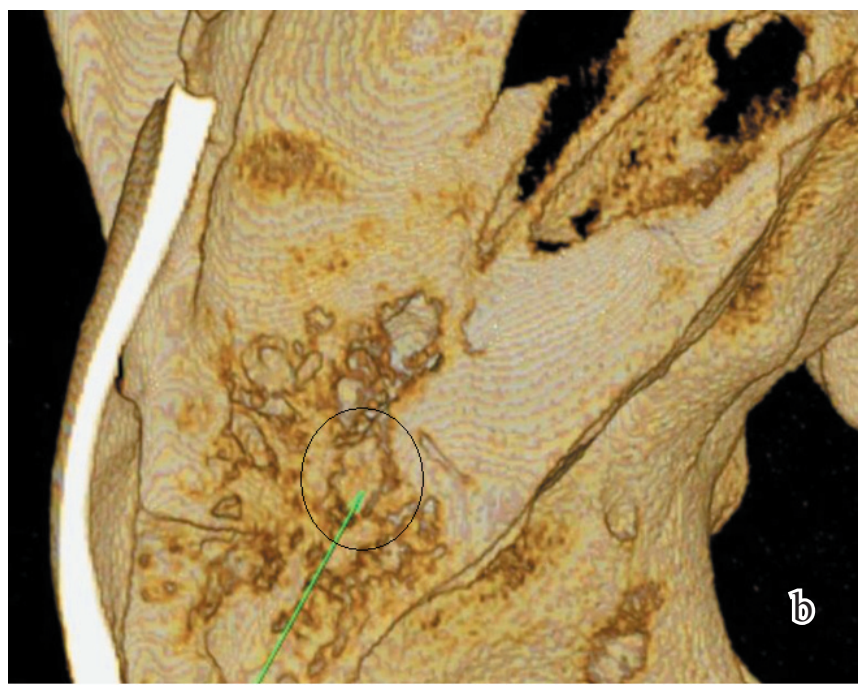

y antro mastoideo izquierdos, con ocupación de oído medio (fig. 4a). En la RM no se observa herniación cerebral. Se realiza una craneotomía temporal izquierda, visualizando un defecto único en el tegmen timpani, que se sella con hueso autólogo, fascia y pegamento biológico (fig. 5). En el postoperatorio, la paciente presenta una afasia de expresión, mostrando la TAC de control un hematoma de lecho

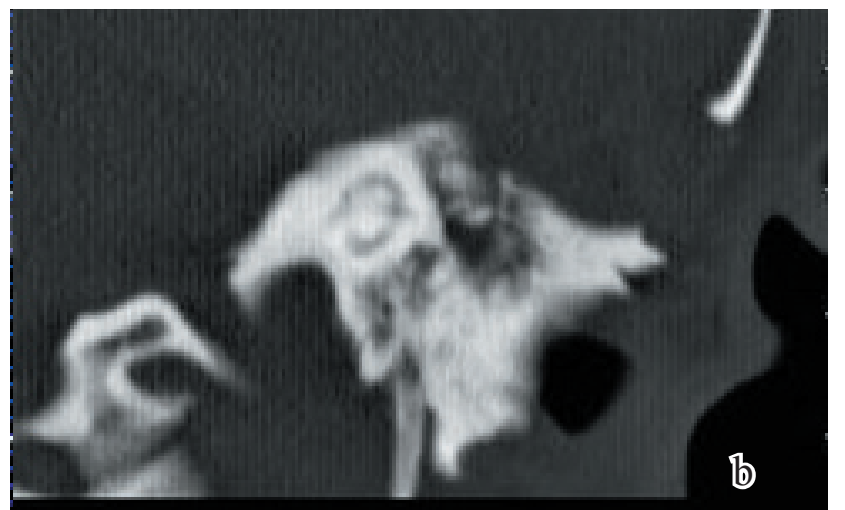

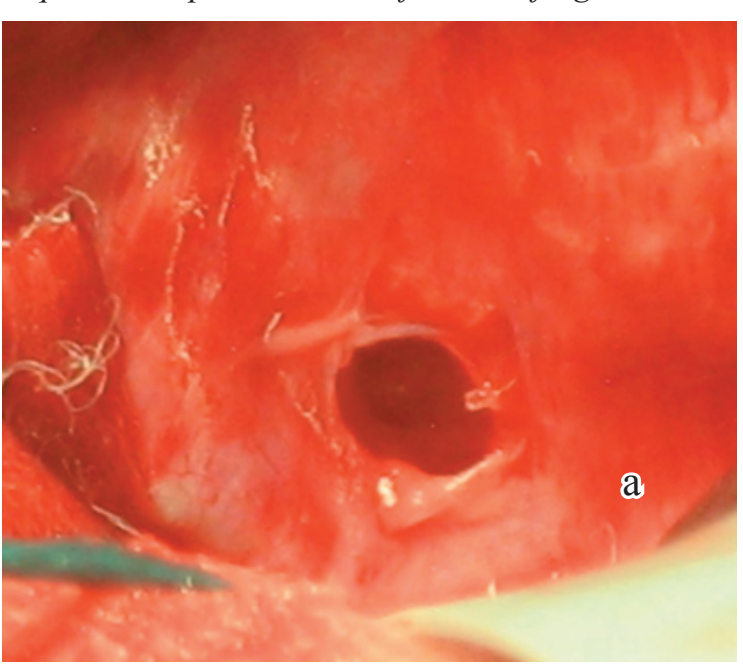

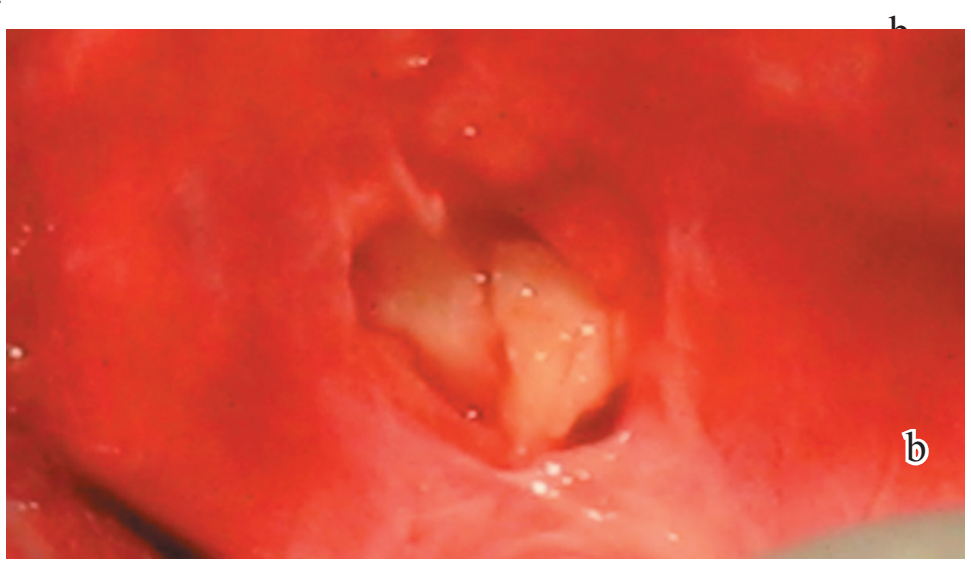

Figura 5. Imágenes intraoperatorias: a) defecto único en tegmen; b) reparación con fragmentos de hueso. 
quirúrgico y un correcto sellado del defecto (fig. 4b). Tras una mejoría progresiva clínica y radiológica, la paciente es dada de alta sin otro dato de focalidad. Vista posteriormente en nuestras consultas, el trastorno del lenguaje es mínimo y no ha vuelto a presentar clínica otológica.

\section{Discusión}

Las fístulas de LCR se dividen clásicamente en adquiridas y espontáneas ${ }^{6}$. La mayoría pertenecen al primer grupo y sus causas más frecuentes son la cirugía basicraneal, los traumatismos craneoencefálicos y la patología crónica inflamatoria o neoplásica ${ }^{8}$. En su estudio sobre 92 pacientes, Savva et $\mathrm{al}^{18}{ }^{18}$, analizaban las fístulas de LCR a través del hueso temporal de cualquier etiología. En el 58\% de los casos, el origen era post-quirúrgico, mientras que en el 32\% había un antecedente de trauma craneal. Sólo en 8 casos (9\%) no se pudo determinar el mecanismo causal, diagnosticándose de fístula espontánea. En la tabla I se analizan algunas de las series más relevantes recogidas en la literatura.

Las fístulas atraumáticas o espontáneas se pueden clasificar, a su vez en dos grupos, según la edad de presentación y su etiopatogenia. En la infancia, el primer signo suele ser un episodio de meningitis en un niño con sordera neurosensorial, ya que en muchos de estos casos asocian defectos congénitos en el hueso temporal ${ }^{17}$. El más frecuente es el que afecta a la cápsula ótica (Neely tipo I), con un laberinto displásico y una alteración de la platina del estribo o de la ventana oval que permiten el escape de LCR. Un ejemplo de ello es la displasia de Mondini, en la que, además de una cóclea y unos conductos semicirculares malformados, puede asociarse un conducto coclear ensanchado y una perforación de la platina del estribo, con el consecuente escape de LCR. Las fístulas perilaberínticas (Neely tipo II) son menos frecuentes y se producen a través de vías óseas preexistentes que no han llegado a sellarse, como el canal petromastoideo, el canal del facial y la fisura de Hyrtl's o timpanomeníngea ${ }^{8}$.

Las fístulas de LCR en el adulto representan el $28 \%$ de los casos espontáneos y obedecen a mecanismos fisiopatológicos diferentes ${ }^{8}$. Una de las teorías más comúnmente aceptada es la de las vellosidades aracnoideas aberrantes, postulada por Gacek et $\mathrm{al}^{7}$. Es bien sabido que un número variable de vellosidades aracnoideas no termina en un seno venoso al final de su desarrollo, y que una vez atravesada la duramadre, queda en contacto con la superficie del hueso. La localización más frecuente de estas vellosidades aberrantes es en la fosa craneal anterior (lateral a la placa cribiforme) y en la fosa craneal media, desde el tegmen timpani hasta la región lateral de la silla turca. Es menos frecuente encontrarlas en la pared posterior del hueso temporal. Con el tiempo, van creciendo y haciéndose más complejas, y asociado al incremento de la actividad física y a la bipedestación, la presión pulsátil del LCR a través de ellas aumenta, siendo capaz de erosionar el hueso. Otra teoría es la que defiende el "origen congénito", que explica las fístulas de LCR a través de aquellas vías óseas preexistentes en la infancia que no han llegado a cerrarse por un desarrollo embrionario anómalo, y que han permanecido clínicamente silentes hasta la etapa adulta ${ }^{3}$. Otros autores, como Ommaya, postulan que áreas de atrofia local en la base del cráneo sobre las que actuaría la presión pulsátil del LCR serían la causa de la fístula ${ }^{14}$.

Dado el alto porcentaje de defectos anatómicos encontrados en hueso de cadáver y la escasa expresividad clínica de esta patología, cabe pensar que deben existir ciertos factores predisponentes para el desarrollo de estas fístulas. Schlosser et $\mathrm{al}^{19}$., estudiando casos de rinolicuorrea espontánea, encontraron que la hipertensión intracraneal era un hallazgo común entre los pacientes, y sugirieron que la obesidad y un elevado índice de masa corporal jugaban también un papel relevante. Otros autores demostraron la asociación entre la obesidad y el síndrome de apnea obstructiva del sueño con las fístulas de $\mathrm{LCR}^{8}$. Prichard et $\mathrm{al}^{16}$., establecieron una relación entre los hallazgos radiográficos de una silla turca vacía (signo altamente correlacionado con una elevada presión intracraneal) y la otolicuorrea espontánea, asociando un índice de masa corporal significativamente superior en estos pacientes comparado con los casos adquiridos. En sus conclusiones, destacan el papel que la hipertensión intracraneal idiopática juega en la patogénesis de las fístulas espontáneas de LCR, y lejos de refutar o sustituir a las teorías previas, establecen una predisposición fisiológica en aquellos pacientes con defectos anatómicos previos ${ }^{16}$. Coppa et $\mathrm{al}^{4}$., recogen un caso de resolución espontánea de siringomielia asociada a Arnold-Chiari tipo I tras la aparición de otolicuorrea espontánea, al reducirse la presión en el espacio subaracnoideo. Todos estos hallazgos obligan a encarar el diagnóstico de esta patología de forma más amplia para identificar factores potencialmente tratables y evitar así su recurrencia.

Numerosos estudios muestran una importante asociación entre las fístulas espontáneas de LCR y el sexo femenino $0^{3,6,7,9,17}$. Suelen ser mujeres de edad media o mayores, generalmente con sobrepeso, y que refieren una historia progresiva de pérdida de audición con sensación de plenitud en el oído, sugestivo de otitis serosa persistente ${ }^{6}$. En muchos casos, es la salida de un líquido claro y constante a través de una miringotomía o la inserción de un tubo de timpanostomía lo que da el diagnóstico ${ }^{6,9}$. La meningitis es la forma de presentación en el $18-25 \%$ de los casos según fuentes de la literatura, siendo menos frecuente que en los niños. Otras manifestaciones, mucho menos habituales, serían los abscesos cerebrales, el pneumoencéfalo o las crisis epilépticas ${ }^{6}$. 


\begin{tabular}{|c|c|c|c|c|c|c|c|}
\hline 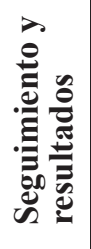 & 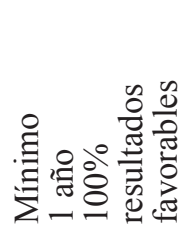 & 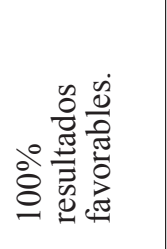 & 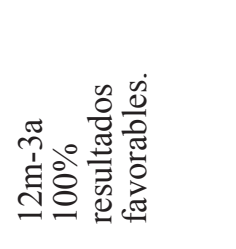 & 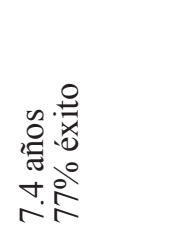 & 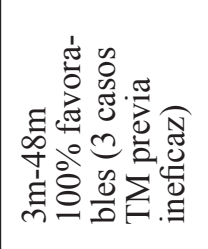 & 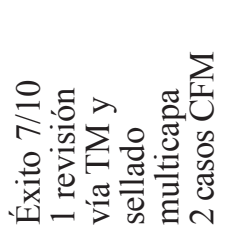 & 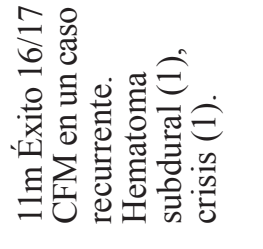 \\
\hline 葛 & 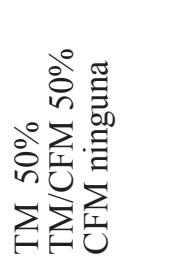 & 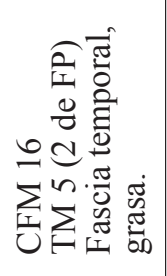 & 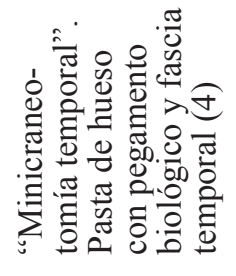 & 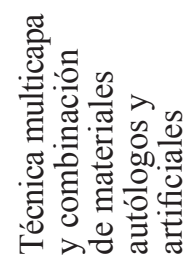 & 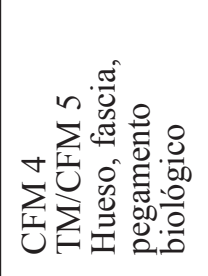 & 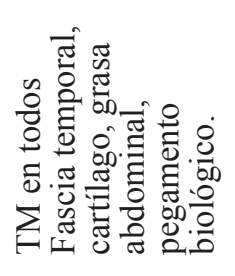 & 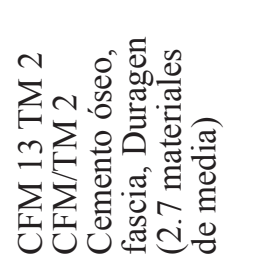 \\
\hline 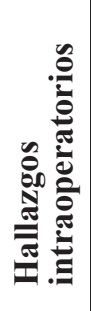 & 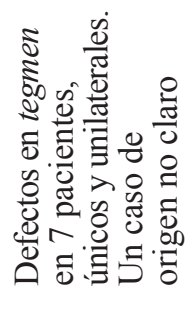 & 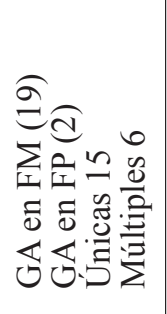 & 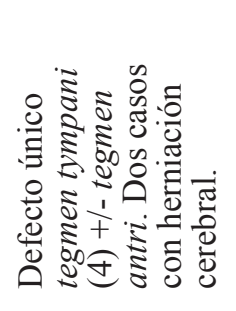 & 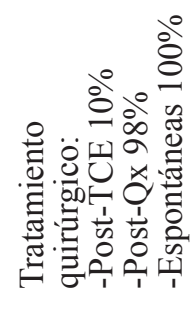 & 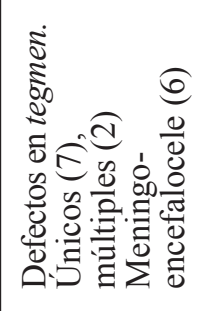 & 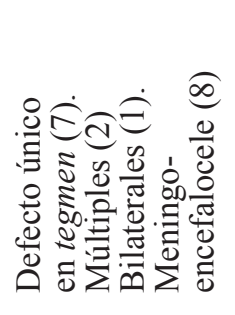 & 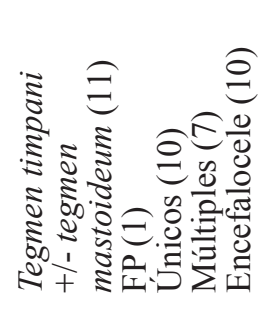 \\
\hline 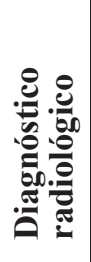 & 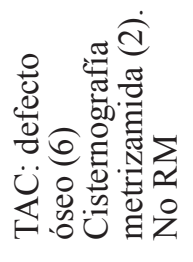 & 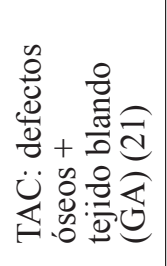 & 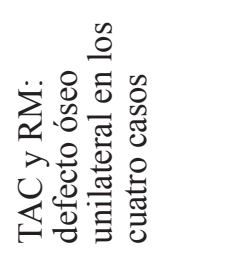 & 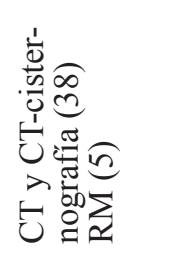 & 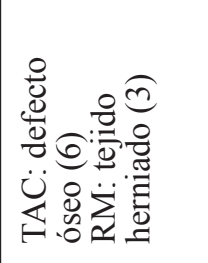 & 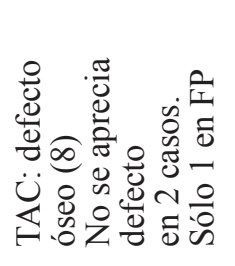 & 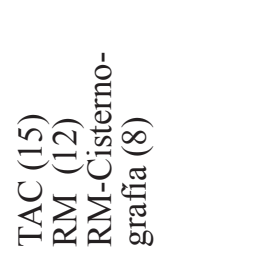 \\
\hline 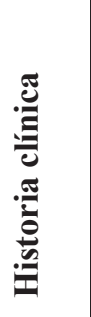 & 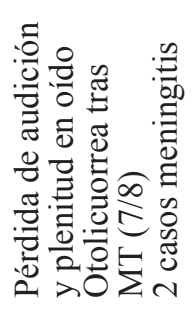 & 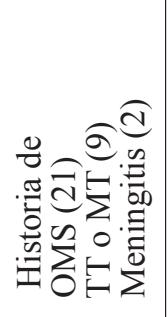 & 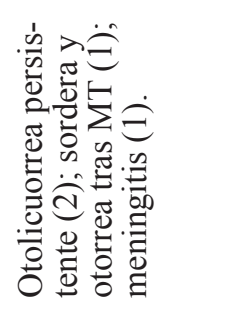 & 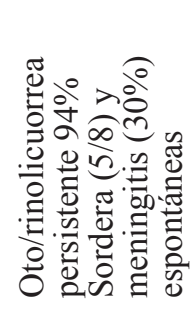 & 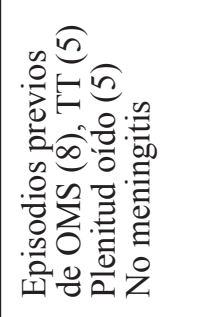 & 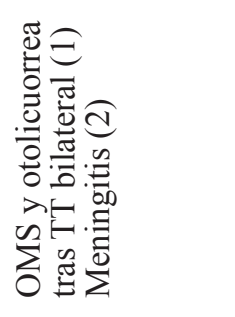 & 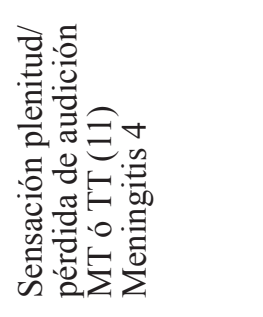 \\
\hline 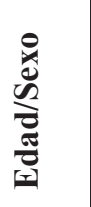 & 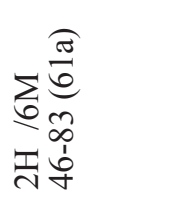 & 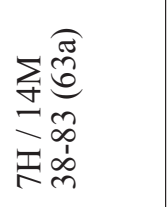 & 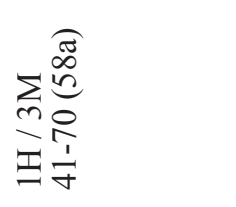 & 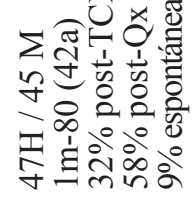 & 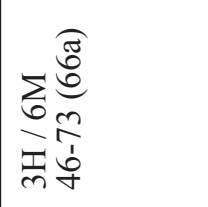 & 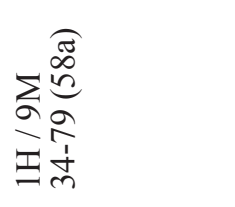 & 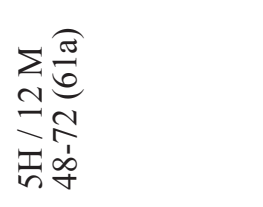 \\
\hline$=$ & $\infty$ & $\vec{\sim}$ & $\nabla$ & ส̆ & $a$ & $\stackrel{ }{ }$ & 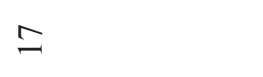 \\
\hline 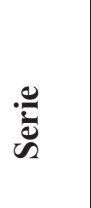 & 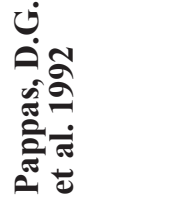 & 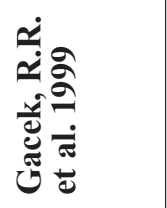 & 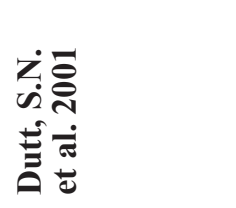 & 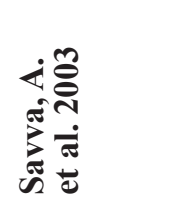 & 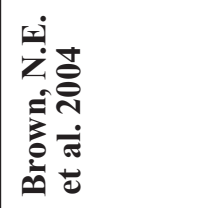 & 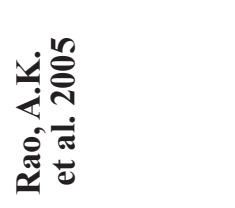 & 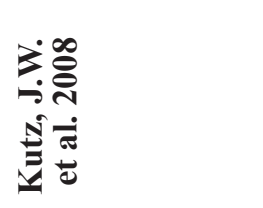 \\
\hline
\end{tabular}


Como ya se ha comentado con anterioridad, una exhaustiva evaluación clínica y un alto índice de sospecha son las claves para un correcto diagnóstico. La exploración física permite identificar las fístulas de LCR hasta en el $90 \%$ de los $\operatorname{casos}^{18}$; la otolicuorrea clara y transparente que se incrementa con maniobras de Valsalva es un dato casi patognomónico ${ }^{6}$, confirmando la naturaleza de LCR con pruebas de laboratorio específicas para ello, como es el análisis de la $\beta$-2-transferrina ${ }^{3}$. Las pruebas de imagen permiten corroborar la sospecha clínica, siendo la TAC del hueso temporal la herramienta más útil ${ }^{18}$. Stone et $\mathrm{al}^{20}$, compararon la eficacia de la TAC de alta resolución sin contraste frente a la cisternotomografía y la cisternografía isotópica. La primera identificaba los defectos óseos en el $71 \%$ de los pacientes, y en todos los casos en los que se procedió a reparar quirúrgicamente la fístula, los hallazgos intraoperatorios se correlacionaban con lo mostrado en la TAC. K. Rao et $\mathrm{al}^{17}$, , recogen resultados similares (sensibilidad del 80\%). La cisternotomografía es defendida por otros autores como la prueba de mayor rendimiento, pero tiene la desventaja de un alto porcentaje de falsos negativos en casos de fístulas intermitentes o de bajo débito.

Los defectos óseos se localizan mayoritariamente en el tegmen timpani y en el tegmen mastoideo ${ }^{3,6,9,17}$, siendo más raros en la pared posterior del hueso temporal, donde el hueso cortical es más grueso y no existen dehiscencias fisiológicas, como en la fosa craneal media. De hecho, la presencia de erosiones en fosa posterior es muy sugerente de la existencia de granulaciones aracnoideas aberrantes, que si bien no suelen tener traducción clínica, deben hacer sospechar una fístula de LCR cuando se acompañan de opacificación de las celdillas mastoideas adyacentes ${ }^{10}$. Los defectos en la fosa craneal media pueden ser únicos o múltiples, uni o bilaterales, y acompañarse o no de herniación de estructuras cerebrales (meningocele o meningoencefalocele). La RM es la prueba de elección para detectar el tejido blando y es complementaria a la $\mathrm{TAC}^{6}$.

El tratamiento de estas fístulas espontáneas de LCR es eminentemente quirúrgico. Como recogieron Savva et $\mathrm{al}^{18}$., en su serie, ninguna de las fístulas atraumáticas se resolvió con técnicas conservadoras, y sólo una de las 53 fístulas post-quirúrgicas curó sin cirugía; sin embargo, el 83\% de los casos postraumáticos cesaron espontáneamente. Dalgic et $\mathrm{al}^{5}$., exponen una alternativa menos invasiva para el manejo de las fístulas de LCR, como es la colocación de un sistema de drenaje lumbar continuo. En su serie de 46 pacientes, con fístulas de origen traumático, consiguieron una tasa de éxito del $88 \%$.

Varios abordajes se han descrito en la literatura para el tratamiento de estas fístulas, siendo dos los principales: la craneotomía de fosa media y la vía transmastoidea, que pueden ser utilizadas solas o en combinación. General- mente se acepta que los defectos del tegmen, sobre todo si son múltiples, grandes o muy anteriores, son más fácilmente reparados mediante craneotomía, mientras que los de fosa posterior se abordan mejor por la vía transmastoidea ${ }^{6}$. Además, el abordaje a la fosa media permite tratar el tejido cerebral herniado y una mejor y más segura colocación del material sellante, minimizando el riesgo de recidiva, a la vez que evita la manipulación de los elementos de la caja timpánica ${ }^{6}$. Sin embargo, es una técnica no exenta de complicaciones, dada la necesidad de manipulación del lóbulo temporal. Algunos autores han tratado de hacer menos invasiva esta cirugía, realizando abordajes con "minicraneotomías", o la keyhole craniotomy ${ }^{12}$.

Así como la vía de abordaje puede ser materia de controversia, la técnica óptima para conseguir el cierre de la fístula no ofrece tantas dudas. Diversos estudios han demostrado que el cierre directo del defecto tiene un riesgo de fracaso mucho mayor que si se refuerza dicho cierre ${ }^{17,18}$. En el estudio de Savva et $\mathrm{al}^{18}$., el refuerzo del cierre primario suponía una tasa de éxito a los dos años del $81 \%$, frente al $42.9 \%$ en caso de no utilizarlo $(p=0.04)$. La diferencia es todavía más significativa si se emplea una técnica multicapa, con un porcentaje de éxito del $100 \%$ a los dos años, frente al 76.2\% si se emplea una técnica simple $(\mathrm{p}<0.01)$.

Los materiales empleados para el cierre de la fístula pueden ser autólogos o artificiales. Hueso cortical, cartílago septal o auricular, fascia lata o temporal y pericráneo son algunos de los materiales autólogos ampliamente utilizados. En su nota técnica, Tender et $\mathrm{al}^{21}$, recogen los buenos resultados obtenidos en 15 pacientes en los que emplearon un colgajo pediculado de músculo temporal interpuesto entre la duramadre y el hueso de la fosa craneal media, sin evidencia de recurrencia de la fístula en un período de seguimiento de más de dos años. El porcentaje de éxito de un cierre multicapa con materiales autólogos llega al 81\%, frente al $50 \%$ si se usan sólo materiales artificiales. Entre ellos, cabría destacar la cera de hueso, Gelfoam, Oxycel, Duragen, la pasta de hueso autólogo ${ }^{6,13}$ o los más recientes cementos de hueso 9 . La combinación de ambos tipos de materiales aumenta la probabilidad de éxito al $100 \%{ }^{18}$. La fijación con sutura de los materiales utilizados o los pegamentos biológicos (Tissue glue, fibrin glue,...) no parecen aportar ventajas significativas ${ }^{18}$.

\section{Conclusiones}

La fístula de LCR espontánea en el adulto es una entidad que debe sospecharse en aquellos casos con otolicuorrea persistente sin antecedente traumático o quirúrgico. El riesgo de meningitis hace indispensable un diagnóstico precoz, mediante una correcta exploración física, la confirmación por el laboratorio de la naturaleza del líquido y una prueba de imagen que corrobore la sospecha clínica. 
El abordaje quirúrgico puede hacerse por vía transmastoidea, si bien la craneotomía de fosa media ofrece una mejor visualización de todo el tegmen y permite una mejor reparación de los defectos óseos. La técnica empleada debe ser un cierre multicapa, que combine materiales autólogos y artificiales para aumentar las probabilidades de éxito.

\section{Bibliografía}

1. Adkins, W.Y. and Osguthorpe, J.D.: Minicraniotomy for management of CSF otorrhea from tegmen defects. Laryngoscope, 1983; 93: 1038-1040.

2. Barcz, D.V.; Raymond, P.W.; Stears, J.; Jafek, B.W.; Shields, M.: Subarachnoid space: middle ear pathways and recurrent meningitis. The American Journal of Otology 1985; 2: 157-163.

3. Brown, N.E.; Grundfast, K.M.; Jabre, A.; Megerian, C.A.; O’Malley, B.W.; Rosenberg, S.I.: Diagnosis and management of spontaneous cerebrospinal fluid-middle ear effusion and othorrea. The Laryngoscope 2004; 114: 800-805.

4. Coppa, N.D.; Kim, H.J.; McGrail, K.M.: Spontaneous resolution of syringomyelia and Chiari malformation type I in a patient with cerebrospinal fluid otorrhea. Journal of Neurosurgery, 2006; 105: 769-771.

5. Dalgic, A.O.; Gezici, H.O.; Dagliolu, A.R.; Akdag, E.; Ergungor, R.: An effective and less invasive treatment of posttraumatic cerebrospinal fluid fistula: closed lumbar drainage system. Minimally Invasive Neurosurgery, 2008; 51: 154157.

6. Dutt, S.N.; Mirza, S.; Irving, R.M.: Middle cranial fossa aproach for the repair of spontaneous cerebrospinal fluid otorrhea using autologus bone pate. Clin. Otolaryngol. 2001; 26: 117-123.

7. Gacek, R.R.; Gacek M.R.; Tart, R.: Adult spontaneous cerebrospinal fluid otorrhea: diagnosis and management. The American Journal of Otology 1999; 20: 770-776.

8. Jégoux, F.; Malard, O.; Gayet-Delacroix, P.B.; Legent, F.; Beauvillain de Montreuil, C.: Hyrtl's fissure: a case of spontaneous cerebrospinal fluid otorrhea. AJNR 2005; 26: 963-966.

9. Kutz, J.W.; Husain, I.A.; Isaacson, B.; Roland, P.S.: Management of spontaneous cerebrospinal fluid otorrhea. The Laryngoscope 2008; 118: 2195-2199.

10. Lee, M.H.; Kim, H.J.; Lee, I.H.; Kim, S.T.; Jeon, P.; Kim, K.H.: Prevalence and appearance of the posterior wall defects of the temporal bone caused by presumed arachnoid granulations and their clinical significance: CT findings.
AJNR 2008; 29: 1704-1707.

11. LeVay, A.J.; Kveton, J.F.: Relationship between obesity, obstructive sleep apnea and spontaneous cerebrospinal fluid otorrhea. The Laryngoscope 2008; 118: 275-278.

12. May, J.S.; Mikus, J.L.; Matthews, B.L.; et al.: Spontaneous cerebrospinal fluid otorrhea from defects of temporal bone: a rare entity? Am J Otol. 1995; 19: 819-823.

13. Moffat, D.A.; da Cruz, M.J.; Batten, A.; Hardy, D.G.: Use of autologous osteocyte containing bone pate for closure of tegmental defects. Am J Otol. 1998; 19: 819-823.

14. Ommaya, A.K.: Cerebrospinal fluid rhinorrhea. Neurology 1964; 15: 106-113.

15. Pappas, D.G.; Hoffman, R.A.; Cohen, N.L.; Pappas Sr., D.G.: Spontaneous temporal bone cerebrospinal fluid leak. The American Journal of Otology 1992; 6: 534-539.

16. Prichard, C.N.; Isaacson, B.; Oghalai, J.S.; Coker, N.J.; Vrabec, J.T.: Adult spontaneous CSF otorrhea: correlation with radiographic empty sella. Otolaryngology-Head and Neck Surgery 2006; 134: 767-771.

17. Rao, A.K.; Merenda, D.M.; Wetmore, S.J.: Diagnosis and management of spontaneous cerebrospinal fluid otorrhea. Otology and Neurotology 2005; 26: 1171-1175.

18. Savva, A.; Taylor, M.J.; Beatty, C.W.: Management of cerebrospinal fluid leaks involving temporal bone: report on 92 patients. The Laryngoscope 2003; 113: 50-56.

19. Schlosser, R.J.; Wilensky, E.M.; Grady M.S., et al.: Spontaneous nasal cerebrospinal leaks and empty sella syndrome: a clinical association. Am J Rhinol 2003; 12: 9196.

20. Stone, J.A.; Castillo, M.; Neelon, B.; Mukherji, S.K.: Evaluation of CSF leaks: high-resolution CT compared with constrast-enhanced CT and radionuclide cisternography. Am J Neuroradiol. 1999; 20: 706-712.

21. Tender, G.C.; Kutz, S.; Awasthi, D.: Vascularized temporalis muscle flap for the treatment of otorrhea. Techincal note. Journal of Neurosurgery, 2003; 98: 1128-1132.

Galbarriatu, L.; Aurrecoechea, J.; Ruiz de Gopegui, E.; Pomposo, I.; Bilbao, G.; González, S.; Undabeitia, J.I.; Novo, J.J.; Aguirrebengoa, K.; Garibi, J.M.: Otolicuorrea espontánea en el adulto. Presentación de dos casos y revisión de la literatura. Neurocirugía 2011; 22: 150-156.

Correspondencia: Lara Galbarriatu Gutiérrez. Servicio de Neurocirugía. Hospital Universitario de Cruces. Plaza CrucesGurutzeta s/n. 48903 Barakaldo Vizcaya. lara.galbarriatugutierrez@osakidetza.net 